\title{
Computerized brain tumor segmentation in magnetic resonance imaging
}

\section{Segmentação computadorizada de tumores do encéfalo em imagens de ressonância magnética}

\author{
Maryana de Carvalho Alegro ${ }^{1}$, Edson Amaro Junior ${ }^{2}$, Rosei de Deus Lopes ${ }^{3}$
}

\begin{abstract}
Objective: To propose an automatic brain tumor segmentation system. Methods: The system used texture characteristics as its main source of information for segmentation. Results: The mean correct match was $94 \%$ of correspondence between the segmented areas and ground truth. Conclusion: Final results showed that the proposed system was able to find and delimit tumor areas without requiring any user interaction.
\end{abstract}

Keywords: Magnetic resonance imaging; Image processing, computerassisted; Brain neoplasms

\section{RESUMO}

Objetivo: Propor um sistema para segmentação automática de tumores do encéfalo. Métodos: 0 sistema emprega parâmetros de textura como sua principal fonte de informação para a segmentação. Resultados: 0s acertos chegaram a $94 \%$ na correspondência entre a segmentação obtida e o padrão-ouro. Conclusão: Os resultados obtidos mostram que o sistema é capaz de localizar e delimitar as áreas de tumor sem necessidade de interação com o operador.

Descritores: Imagem por ressonância magnética; Processamento de imagem assistida por computador; Neoplasias encefálicas

\section{INTRODUCTION}

Magnetic resonance imaging (MRI) is important tools in brain tumor treatment because they provide a noninvasive method to visualize brain internal structures with high anatomical resolution. The MRI is usually the technique of choice for tumor growth assessment, surgery planning, and chemotherapy and radiotherapy follow-up ${ }^{(1,2)}$. The segmentation of the tumor area allows to quantity the mass volume and its response to the treatment, but it usually has to be performed manually, what is a time-consuming and cumbersome activity that yields two main problems ${ }^{(3)}$ : (1) low response to small changes and (2) high variability between different segmentations. Computer systems can ease the manual segmentation burden while providing a tool for easily assessing tumor mass development ${ }^{(4,5)}$.

This study presents a brain tumor segmentation method for MRI which purpose is to easily segment tumor masses in MRI images with reproducible results. Our pipeline is capable of automatically segment tumor masses, which means that there is no need for manual selection of a starting region, unlike semi-automatic segmentation schemes. We use texture analysis techniques for extracting useful information that allow the discrimination between normal and tumor tissues. Textures features were chosen because it could detect image patterns often inconspicuous to the human eye ${ }^{(6)}$; such ability can be very handy in detecting the subtle MRI signal changes induced by the presence of abnormal tissue. Texture analysis has a long history in medical imaging, and several studies have used $\mathrm{it}^{(7-9)}$.

On the following sections we describe a computational pipeline and the empirical results accomplished. Lastly, the pro and con of the system are discussed.

\footnotetext{
Study carried out at Electrical Engineering Department, Escola Politécnica, Universidade de São Paulo - USP, São Paulo (SP), Brazil.

Integrated System Laboratory, Escola Politécnica, Universidade de São Paulo - USP, São Paulo (SP), Brazil; Instituto do Cérebro - InCe, Hospital Israelita Albert Einstein - HIAE, São Paulo (SP), Brazil.

2 Department of Diagnostic Imaging and Instituto do Cerebro - InCe, Hospital Israelita Albert Einstein - HIAE, São Paulo (SP), Brazil.

${ }^{3}$ Integrated System Laboratory, Escola Politécnica, Universidade de São Paulo - USP, São Paulo (SP), Brazil.

Corresponding author: Maryana de Carvalho Alegro - Instituto do Cérebro, InCe - Avenida Albert Einstein, 627, $2^{\circ}$ subsolo, bloco A - Morumbi - Zip code: $05651-901$ - São Paulo (SP), Brazil -

E-mail: maryana@lsi.usp.br

Received on: Feb 23, 2012 - Accepted on: May 14, 2012
}

Conflicts of interest: none. 


\section{OBJECTIVE}

To propose a brain tumor segmentation system suited for MRI processing, which purpose is to easily segment tumor masses in MRI with reproducible results.

\section{METHODS}

The study was structured as a pipeline of four main stages: pre-processing, feature computation,

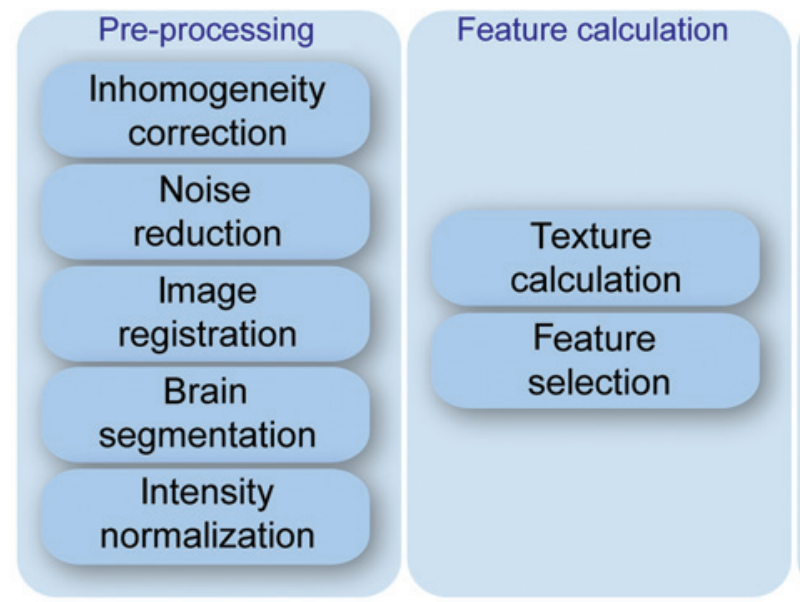

Figure 1. Segmentation pipeline

\section{Pre-processing}

MRI suffer from several artifacts inherent to the acquisition process and chemical composition of the underlying tissues. The pre-processing stage is responsible for reducing such artifacts to improve the segmentation results.

Field inhomogeneity is one of the most know MRI artifacts. It is caused by small magnetic fluctuations in the main static field, poor radio frequency (RF) coil performance, eddy currents, motion blur, among other causes. Inhomogeneity manifests itself as a smooth intensity variation field that spans the entire image. The scanner calibration alone is not enough to correct this artifact; so image-enhancing techniques must be applied to reduce inhomogeneity effects. Intensity inhomogeneity is corrected in the first step of the pre-processing stage (Figure 1) using the N3 algorithm ${ }^{(10)}$. The algorithm creates estimated distributions for the real image intensities and corruptive field, which causes the inhomogeneity, based on the underlying image. These estimates are then used to correct the original image.

Noise is another well-known MRI artifact that corrupts image information and degrades computer analysis results. The main noise causes are poor RF segmentation, and post-processing. This pipeline works with three different types of MRI: T1, T1 with contrast (T1c) and FLAIR images. Since each modality is better suited for imaging a different type of tissue (i.e. FLAIR for edema and T1 for fat), they are handled as separated information channels. Thus, all pipeline stages are designed to work with the three MRI modalities at the same time. The pipeline and its stages are illustrated in figure 1.

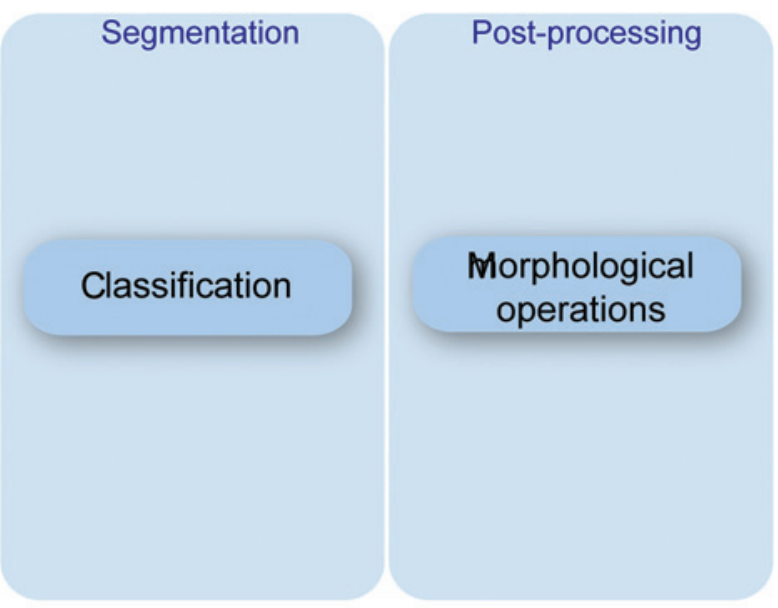

shielding, which causes external RF interference to reach the scanner, and thermal effects that occurs inside the patient's body. The second step of the preprocessing stage is responsible for noise reduction, which is performed by the anisotropic diffusion ${ }^{(11)}$. This algorithm is a well-known general-purpose noise filtering method, which smooths homogeneous areas of the image whereas enhancing the borders.

After the two previous steps, T1, T1c and FLAIR MRI must be transformed to the same coordinate system, so that the same anatomical structures present in all images are aligned. This allowed the pipeline to operate simultaneously over all "channels". Our pipeline currently uses a semi-automatic algorithm based on local means ${ }^{(12)}$, which requires the manual selection of control points. Nevertheless, due to the modular formulation of the pipeline this can be easily replaced by an automated method.

The brain segmentation step is responsible for separating the brain from adjacent regions, such as meninges and skull bones; and from background noise. The disposal of unnecessary pixels speeds up the overall pipeline performance and keeps the process focused on the pieces of image that have important information. Our pipeline uses a method adapted 
from the studies of Brummer et al. ${ }^{(13)}$ and Shen et al. ${ }^{(14)}$, which creates a gross segmentation of the brain area. This mask initializes an active-contours segmentation algorithm $^{(15)}$ that performs the final segmentation.

Unlike other medical imaging techniques, such as X-ray and computerized tomography, the MRI pixel intensities have no fixed value on the imaged tissue. So, pixels depicting the same tissue can have different intensities in adjacent slices, what degrades computational analyses that are performed directly on the intensity values. This artifact is called intensity non-standardization and we attempt to reduce it applying a normalization procedure ${ }^{(16,17)}$ in our last pre-processing step.

\section{Feature calculation}

In image processing studies, texture can be characterized as the spatial distribution and spatial dependency between pixels of an image. Texture analysis is a set of techniques that aim to mathematically characterize these distributions and dependencies. Texture features can be roughly separated in four different groups: statistical, structural, model-based and transform-based.

Structural methods are commonly used in image synthesis but seldom in image analysis, thus are not further discussed. Statistical texture analysis methods use elements from probability and statistics theory, such as histogram and probability density functions, to quantify the texture present in an image. Modelbased methods rely on existing mathematical models, which are assumed to rule texture shaping; and their parameters are regarded as image features. Finally, transform-based methods rely on the assumption that image spectra carries important information about the texture content, thus spectral coefficients are used as texture features ${ }^{(18)}$ for an in-depth overview of texture analysis.

This pipeline stage calculates statistical features as co-occurrence texture features ${ }^{(19,20)}$ (angular second moment, contrast, correlation, variance, inverse difference moment, sum average, sum variance, entropy, sum entropy, difference variance, difference entropy) and run-length features ${ }^{(21,22)}$ (SRE, LRE, GLN, RLN, RP, LGRE, HGRE, SRLGE, SRHGE, LRLGE, LRHGE). All these features are calculated for $0^{\circ}, 45^{\circ}, 90^{\circ}$ and $135^{\circ}$ occurrence angles. It also calculates model-based features (fractal dimension ${ }^{(23)}$ and Markov random fields) ${ }^{(24)}$ and transform-based features $(\mathrm{LH}, \mathrm{HH}$ and HL channels for two levels of Daubechies 4, coiflets 3 , symlets 2 and biortogonais 5.5 wavelets and a bank with six different Gabor filters ${ }^{(25)}$ ). These features are calculated for each MRI modality (T1, T1c and FLAIR), yielding a total of 477 features.

The resulting feature set is large and may carry useless information that can degrade the segmentation process and increase computational time. Thus, a feature selection algorithm ${ }^{(26)}$ is applied to select the most informative features. Only the ten most important features were taken into account.

\section{Segmentation}

Brain MRI have a complex structure for which basic image processing segmentation techniques hardly produces good results. To improve performance more "clever" algorithms are needed for analyzing information derived from these images.

We chose to use a machine learning technique called Support Vector Machine (SVM) ${ }^{(27)}$ to classify pixels in either tumor or normal, based solely on the texture feature calculated in the previous stage. The SVM is a supervised learning method, meaning that the algorithm needs to be trained. Training takes place by presenting a set of manually labeled data (a pair $(\mathrm{X}, \mathrm{y})$, where $\mathrm{X}$ is the vector of texture features calculated over one pixel and y its respective class 1 for tumor and 0 for normal) to the classifier that "learns" from this set how a tumor/normal pixel should behave (mathematically speaking, the SVM calculates a separating hyperplane over the feature space and uses this information to classify unknown data). Segmentation per se is done by applying the trained classifier on the information calculated from each non-background pixel of the MRI images (the aforementioned texture features). This process results in a mask of the tumor area.

\section{Post-processing}

The classifier may assign wrong labels to some pixels, creating spurious connected regions in the segmented area and leading to an imperfect segmentation. To refine the final result, a morphological erosion operation is applied to segmented region obtained in the previous stage. This removes small lumps and smooths the result, creating a big connected region (mask) corresponding to the tumor area, but it might also create unwanted holes in the mask. Any existing hole inside this region is closed and morphological dilation is applied for further refinement. The final result is a smooth roundish mask corresponding to the tumor area. 


\section{System development and result assessment}

The pipeline used was developed in MATLAB (Mathworks, USA), which allowed faster system prototyping. The inhomogeneity correction in the preprocessing stage was done with the MIPAV tool ${ }^{(28)}$. The system was tested with image sets from 11 patients (numbered from \#1 to \#11). Images were obtained from the PACS system in anonymized DICOM format at medical school of the Universidade de São Paulo (USP). Tests were conducted using tree adjacent slices from each modality (T1, T1c and FLAIR) of each patient. The patient set contained image examples of several tumor types. Patient details can be seen in table 1.

Table 1. Patient information. Tumor types were histopathologically confirmed

\begin{tabular}{lccc}
\hline Patient & Gender & Tumor type & Age (years) \\
\hline$\# 1$ & Female & Meningioma & 36 \\
$\# 2$ & Female & GBM & 64 \\
$\# 3$ & Female & Medulloblastoma & 26 \\
$\# 4$ & Male & GBM & 63 \\
$\# 5$ & Female & Meta-carcinoma & 73 \\
$\# 6$ & Male & Pnet & 3 \\
$\# 7$ & Male & Astrocytoma grade II & 79 \\
$\# 8$ & Male & GBM & 63 \\
$\# 9$ & Male & GBM & 56 \\
$\# 10$ & Female & Ependymoma & 17 \\
$\# 11$ & Male & Astrocytoma grade II & 52 \\
\hline
\end{tabular}

\section{RESULTS}

Two experienced radiologists (here called GT\#1 and GT\#2) manually segmented the tumor masses in the MRI images used for testing. These masks were considered the ground truth and were used for assessing the system efficiency. The classifier was trained in the leave-one-out scheme, in which to each iteration a case was left out of the training phase, that was performed with the remaining data. This process was repeated until all cases were used. The ground truth masks were used in the SVM training process. The leave-one-out process was repeated twice by each physician. Some of the segmentation and results physician masks are illustrated in figure 2 .

The quantitative results were calculated using a measure of percentage match between ground truth and the segmentation result ${ }^{(29)}$. Therefore, TP is the set of true positive pixels and GT is the set of ground truth pixels. The symbol \#(.) stands for the number of elements in the set. We also used a correspondence ratio

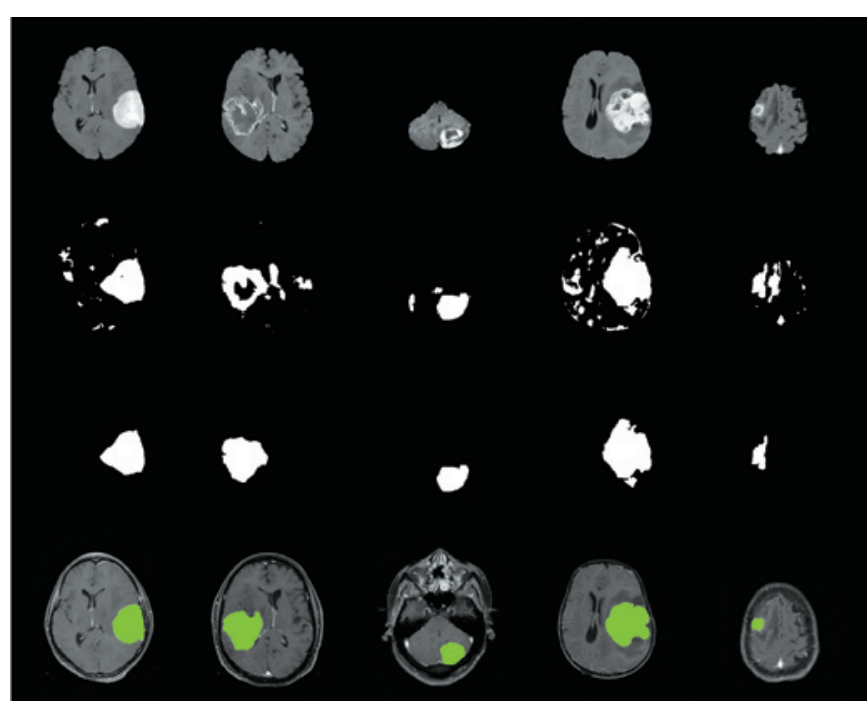

Figure 2. Pre-processed T1c MRI slices of five different cases can be seen in the first row of the image. The second row displays their respective segmentation results before post-processing. The third row shows the final segmentation results and the last row shows the ground truth (green) drawn by physician \# 1 on top of the original magnetic resonance imaging

(CR) defined as follow ${ }^{(29)}$ : FP is the set of false positive pixels. Higher percent match (PM) values indicate the existence of a greater number of true positive pixels inside the segmented area. The $\mathrm{CR}$ value assesses how far the segmentation is from the ground truth. The ideal $\mathrm{CR}$ value is 1 , which indicates a PM between ground truth and segmentation. $\mathrm{CR}$ values closer to 1 indicates that the segmentation holds more true positives than false positives, while negative $\mathrm{CR}$ values indicates the existence of more false positives than true positives in the segmentation.

Table 2. Case indicates the patient number inside

\begin{tabular}{|c|c|c|c|c|c|c|}
\hline \multirow{2}{*}{ Case } & \multicolumn{2}{|c|}{ GT \#1 } & \multicolumn{2}{|c|}{ GT \#2 } & \multicolumn{2}{|c|}{ Mean } \\
\hline & PM & CR & PM & CR & PM & CR \\
\hline$\# 1$ & 99.05 & 0.82 & 99.39 & 0.82 & 99.22 & 0.82 \\
\hline \#2 & 88.66 & 0.75 & 89.40 & 0.78 & 89.03 & 0.76 \\
\hline \#3 & 91.03 & 0.11 & 96.74 & 0.25 & 93.88 & 0.18 \\
\hline \#4 & 99.93 & -1.41 & 99.84 & -1.33 & 99.89 & -1.26 \\
\hline$\# 5$ & 99.34 & -2.54 & 99.28 & -1.38 & 99.31 & -1.96 \\
\hline \#6 & 99.91 & 0.71 & 99.44 & 0.72 & 99.67 & 0.72 \\
\hline$\# 7$ & 96.04 & 0.59 & 80.43 & 0.66 & 88.23 & 0.62 \\
\hline \#8 & 99.76 & 0.80 & 99.54 & 0.80 & 99.65 & 0.79 \\
\hline \#9 & 95.03 & 0.82 & 88.79 & 0.80 & 91.91 & 0.81 \\
\hline$\# 10$ & 98.58 & -0.06 & 99.49 & -0.83 & 99.03 & -0.44 \\
\hline \multirow[t]{2}{*}{ \#11 } & 58.57 & -1.07 & 89.82 & -1.94 & 74.20 & -1.51 \\
\hline & & & & Total mean & 94.00 & -0.04 \\
\hline
\end{tabular}

GT\#1 and GT\#2 indicates dado test with masks drawn by physician \#1 and \#2 respectively. PM stands for the percentage match measure and $\mathrm{CR}$ for the correspondence ratio. 
Results of individual tests of each case can be seen in table 2. Our tests achieved 94\% mean PM and -0.04 mean CR. The high PM value indicated good classification accuracy but the mean negative CR indicated the existence of overestimation of the tumor areas (greater number of false positives).

\section{DISCUSSION}

This study aimed to design, implement and evaluate a system for brain tumor segmentation in clinical practice MRI. Our system was formulated as a pipeline composed of four main stages and texture information was in its core. Our results suggested that the proposed system is able to handle the complexity inherent to brain MRI.

The lack of a trustworthy ground truth was the main obstacle faced in this study. Only histopathological analysis can fully assess the borders of a tumor mass, especially for infiltrating types like the astrocytoma. Nevertheless, by the time we performed our investigation, there were no publicly available databases, which contained both MRI and histological information. Hence, we had to compare our segmentation results against masks manually drawn by experienced radiologists. Such problem may have introduced some uncertainty in our results, because it is impossible to make a true evaluation without knowledge about the real tumor borders.

Nonetheless, our system did remarkably well, achieving $94 \%$ mean percent match between automatic segmented areas and ground truth. However, the negative mean $\mathrm{CR}$ value indicated the existence of overestimated tumor area. A look at the individual result showed that most of the cases had a good segmentation performance (high PM and high positive CR values) but four cases presented severe overestimation of the tumor area. Since we did not verify histologically the ground truth, we could not assert whether it was a imperfection in our system method or if the system actually captured invisible tissue differences in the tumor adjacencies.

\section{CONCLUSION}

These results showed that the proposed system was able to locate and delimit tumor areas without requiring any user interaction.

\section{ACKNOWLEDGEMENTS}

We thank Dr. Rita de Cássia Pincerato, from the Hospital das Clínicas, of the Universidade de São Paulo, for providing the patient images; and Dr. Claudia da Costa Leite, for patiently drawing part of the golden standard segmentations used in the results section.

\section{REFERENCES}

1. Moore AJ, Newell DW, Lumley J. Neurosurgery principles and practice. New York: Springer; 2004.

2. Ali-Osman F. Brain tumors. Totowa, NJ: Humana Press; 2005.

3. Rangayyan RM. Biomedical image analysis. Florida: CRC; 2005.

4. Clarke LP, Velthuizen RP, Clark M, Gaviria J, Hall L, Goldgof D, et al. MRI measurement of brain tumor response: Comparison of visual metric and automatic segmentation. Magn Reson Imaging. 1998;16(3):271-9

5. Vaidyanathan M, Clarke LP, Hall LO, Heidtman C, Velthuizen R, Gosche K, et al. Monitoring brain tumor response to therapy using MRI segmentation. Magn Reson Imaging. 1997; 15(3):323-34.

6. Zhang J, Ma KK, Er MH. Tumor segmentation from magnetic resonance imaging by learning via one-class support vector machine [Internet]. In: International Workshop on Advanced Image Technology 2004 [cited 2012 Apr 12]. Available from: http://lear.inrialpes.fr/people/zhang/IWAITO4.pdf

7. Zhang Y, Zhu H, Mitchell JR, Costello F, Metz LM. T2 MRI texture analysis is a sensitive measure of tissue injury and recovery resulting from acute inflammatory lesions in multiple sclerosis. Neurolmage. 2009;47(1):107-11.

8. Bernasconi A, Bernasconi N, Caramanos Z, Reutens DC, Andermann F, Dubeau F, et al. T2 relaxometry can lateralize mesial temporal lobe epilepsy in patients with normal MRI. Neurolmage. 2000;12(6):739-46.

9. de Oliveira MS, Balthazar ML, D’Abreu A, Yasuda CL, Damasceno BP, Cendes $\mathrm{F}$, et al. MR imaging texture analysis of the corpus callosum and thalamus in amnestic mild cognitive impairment and mild Alzheimer disease. AJNR Am J Neuroradiol. 2011;32(1):60-6.

10. Sled JG, Zijdenbos AP. A nonparametric method for automatic correction of intensity nonuniformity in MRI data. IEEE Trans Med Imaging. 1998;17(1):87-97.

11. Perona P, Malik J. Scale-space and edge detection using anisotropic diffusion. IEEE Trans Pattern Anal Mach Intell. 1990;12(7):629-39.

12. Gonzalez RC, Woods RE, Eddins SL. Digital image processing using MATLAB. New Jersey: Pearson Prentice Hall; 2004.

13. Brummer ME, Mersereau RM, Eisner RL, Lewine RJ. Automatic detection of brain contours in MRI data sets. IEEE Trans Med Imaging. 1993;12(2):153-66.

14. Shen S, Sandham WA, Granat MH. Preprocessing and segmentation of brain magnetic resonance images. In: $4^{\text {th }}$ International Conference IEEE Special Topic Conference on Information Technology Applications in Biomedicine; 2003 April 24-26. p.149-52.

15. Chan TF, Vese LA. Active contours without edges. IEEE Trans Image Process. 2001;10 (2):266-77.

16. Nyúl LG, Udupa JK. On standardizing the MR image intensity scale. Magn Reson Med. 1999;42(6):1072-81

17. Nyúl LG, Udupa JK, Zhang X. New variants of a method of MRI scale standardization. IEEE Trans Med Imaging. 2000;19(2):143-50.

18. Petrou M, Sevilla PG. Image processing: dealing with texture. Chichester: Wiley; 2006.

19. Haralick RM. Statistical and structural approaches to texture. Proc IEEE Inst Electr Electron Eng.1979;67(5):786-804.

20. Haralick RM, Shanmugam K, Dinstein I. Textural features for image classification. IEEE Trans Syst Man Cybern.1973;3(6):610-21.

21. Chu A, Sehgal CM, Greeleaf JF. Use of gray value distribution of run lengths for texture analysis. Pattern Recognit Lett. 1990;11(6):415-9.

22. Dasarathy BV, Holder EB. Image characterizations based on joint gray levelrun length distributions. Pattern Recognit Lett. 1991;12(8):497-502.

23. Pentland AL. Fractal-based description of natural scenes. IEEE Trans Pattern Anal Mach Intell. 1984;6(6):661-74. 
24. Manjunath BS, Chellappa R. Unsupervised texture segmentation using Markov random field models. IEEE Trans Pattern Anal Mach Intell. 1991;13(5):478-82.

25. Zacharaki El, Wang S, Chawla S, Soo Yoo D, Wolf R, Melhem ER, et al. Classification of brain tumor type and grade using MRI texture and shape in a machine learning scheme. Magn Reson Med. 2009;62(6):1609-18.

26. Peng $H$, Long F, Ding $C$. Feature selection based on mutual information criteria of max-dependency, max-relevance, and min-redundancy. IEEE Trans Pattern Anal Mach Intell. 2005;27(8):1226-38.
27. Lorena $A C$, Carvalho $A C$. Introdução às máquinas de vetores de suporte. RITA. 2003;14(2):43-67.

28. National Institutes of Health. Center for Information Technology. About MIPAV [Internet]. 2007 [cited 2012 Apr 12]. Available from: http://mipav.cit. nih.gov/

29. Zhou J, Chan KL, Chong VF, Krishnan SM. Extraction of brain tumor from MR images using one-class support vector machine. Conf Proc IEEE Eng Med Biol Soc. 2005;6:6411-4. 